REVISTA 89.1

Revista RELACIONES INTERNACIONALES

Escuela de Relaciones Internacionales.

Universidad Nacional, Costa Rica.

N. ${ }^{\circ} 89.1$ • Enero-Junio de 2016

doi: http://dx.doi.org/10.15359/ri.89-1.7

Pp. 151-169

\title{
EL DESARROLLO DEL SECTOR DE SOFTWARE EN AMÉRICA LATINA Y ASIA
}

\author{
Rolando Javier Salinas García* \\ Daniel Montes Pimentel**
}

\section{RESUMEN:}

Organizaciones Internacionales como la OCDE, la CEPAL, la ONU y el FMI se han pronunciado a favor del desarrollo del sector de las Telecomunicaciones (TIC) en los países emergentes, para generar desarrollo económico, y por consiguiente, desarrollo social. Ante esta tendencia, el surgimiento de los conceptos de economía y sociedad del conocimiento sirven para re-pensar a la sociedad basada en la creación y el flujo intensivo de conocimiento. Por lo que los nuevos parámetros de desarrollo se han enfocado en los indicadores de acceso al internet, el uso de tecnologías en la sociedad, empresas de alta tecnología e instituciones educativas. El presente artículo versa sobre una comparación entre México, Uruguay, China y la República de Corea (Corea de Sur), específicamente sobre cómo han desarrollado el sector del software y la forma en cómo se han insertado en el mercado global del software.

Palabras clave: sector de software, economía del conocimiento, sociedad del conocimiento, mercado global del software.

\begin{abstract}
:
International Organizations such as OCDE, CEPAL, the UN and the IMF have spoken in favor of the development of Telecommunications in developing countries to generate economic and social development. Under this trend, the emergence of the concepts of Knowledge Economy and Knowledge Society serves as an opportunity to imagine a new kind of society based on the creation of knowledge as the main competitive advantage. The new parameters of development focus on indicators of access to the Internet, the use of technology in society, hightech firms and educational institutions. This article is a comparison between Mexico, Uruguay, China and the Republic of Korea, specifically how these countries have developed their software sector and the actions taken to participate in the global software market.
\end{abstract}

Keywords: Software Sector; Knowledge Economy; Knowledge Society; Software Global Market.

* Profesor/Investigador de la Universidad Autónoma de Querétaro. Coordinador de la Unidad Multidisciplinaria de Estudios sobre el Trabajo (UMEST) Campus UAQ-Aeropuerto. Correo electrónico: javier.salinas.uaq@gmail.com

** Licenciado en Piscología Social y Maestro en Estudios Multidisciplinarios sobre el Trabajo por la Universidad Autónoma de Querétaro (UAQ). Correo electrónico: danielmontes.uaq@gmail.com 


\section{Introducción}

Las TIC, a nivel mundial, se han colocado como la panacea del desarrollo y la transformación social, debido a que las nuevas tecnologías desarrolladas ayudan a mejorar la calidad de vida y facilitan la reestructuración productiva. Con esta tendencia, ninguna región del mundo se ha quedado fuera de esta lógica y diferentes países están desarrollando políticas para lograr esta transformación.

En los años noventa, Israel, India, Irlanda, Colombia, Costa Rica, Chile, Argentina, Sudáfrica, Brasil, Uruguay; entre otros países, se consideraban grandes promesas para incorporarse al mercado global de software. Hoy la India, la República de Corea (Corea del Sur), Brasil y Uruguay han permanecido en el mercado global de software logrando reputación para sus recursos humanos y empresas locales. Por su parte, China y México se han posicionado como nuevos espacios de desarrollo y países consumidores de TIC. Este panorama invita a reflexionar sobre la forma en que se ha desarrollado el sector de software y por qué regiones como Asía ha tenido un desarrollo agigantado; mientras América Latina encuentra un avance lento. La importancia de hacer comparaciones entre estas dos regiones se centra en la transformación mundial a partir del crecimiento de los países asiáticos y la innovación desarrollada en los últimos años que modifican al mercado global de software; además, que China y La República de Corea se han convertido en grandes competidores y consumidores de TIC.

\section{El sector del software en China, República de Corea, Uruguay y México}

El Banco Mundial pública una comparación de los países afiliados para analizar la infraestructura para el desarrollo de las TIC en cada país. La República de Corea se ha convertido en el país mejor rankeado (19) y supera en demasía a México; por su cuenta, China ha tenido un gran desarrollo que le ha permitido superar a México y estar detrás de La república de Corea en los países con mejor infraestructura de TIC. 


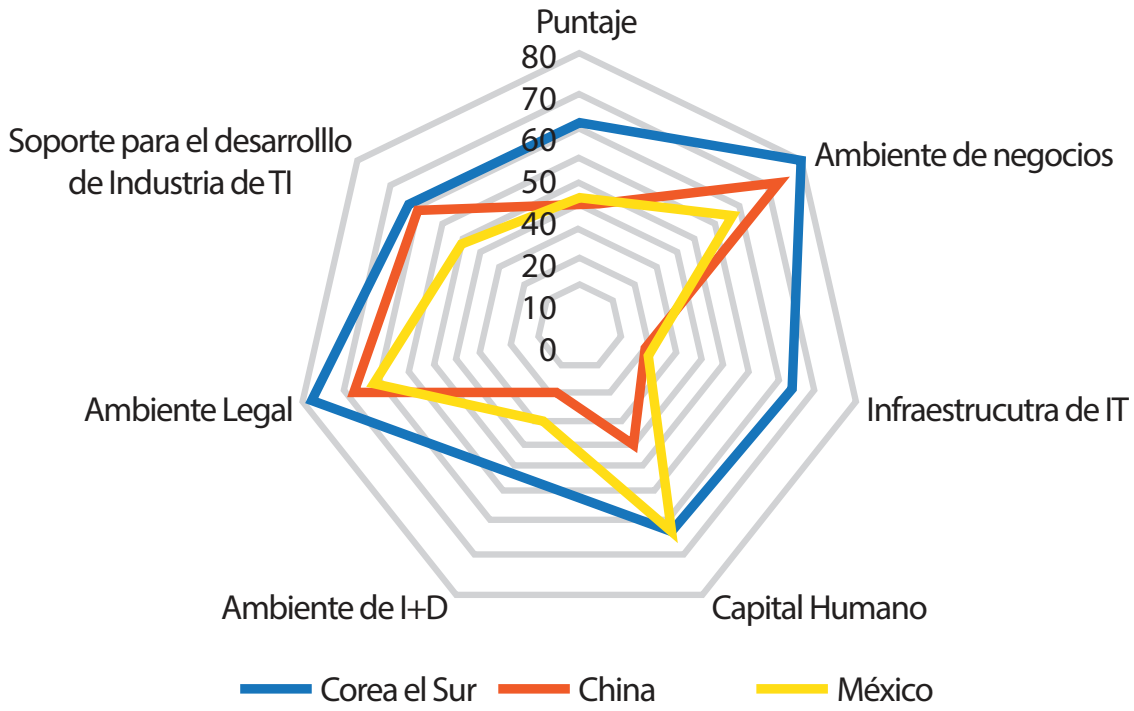

Figura 1. Gráfico comparativo de ambiente de TIC entre países.

Elaboración propia con base en The Global Information Technology Report (2014).

La consolidación del sector del software se basa en la transformación social hacía una sociedad del conocimiento, "idealmente, la sociedad del conocimiento presupone no sólo una sociedad alfabetizada o alfabetizada en computadora, o incluso una sociedad educada, sino más bien una sociedad altamente educada, que demanda no precisamente trabajadores calificados, sino trabajadores del conocimiento" (Majumdar, 1998, citado en Casas y Dettmer, 2013, p. 14). Esta dinámica coloca al recurso humano como parte importante para la consolidación y el desarrollo de software. Uruguay y México están por debajo de la media de la OCDE en habilidades lectoras, matemáticas y ciencia. La diferencia entre los países de América Latina y los países asiáticos es de más de 100 puntos. La dinámica de Asía en los últimos años generó un gran nivel de competitividad, lo que ha obligado a los países asiáticos a mejorar el nivel educativo; ejemplo de esto es que dentro de los primeros diez lugares en la prueba PISA, siete lugares son ocupados por países asiáticos. Esto deja en claro que los sectores intensivos en conocimiento pueden consolidarse a través de una educación de calidad desde la enseñanza básica. 


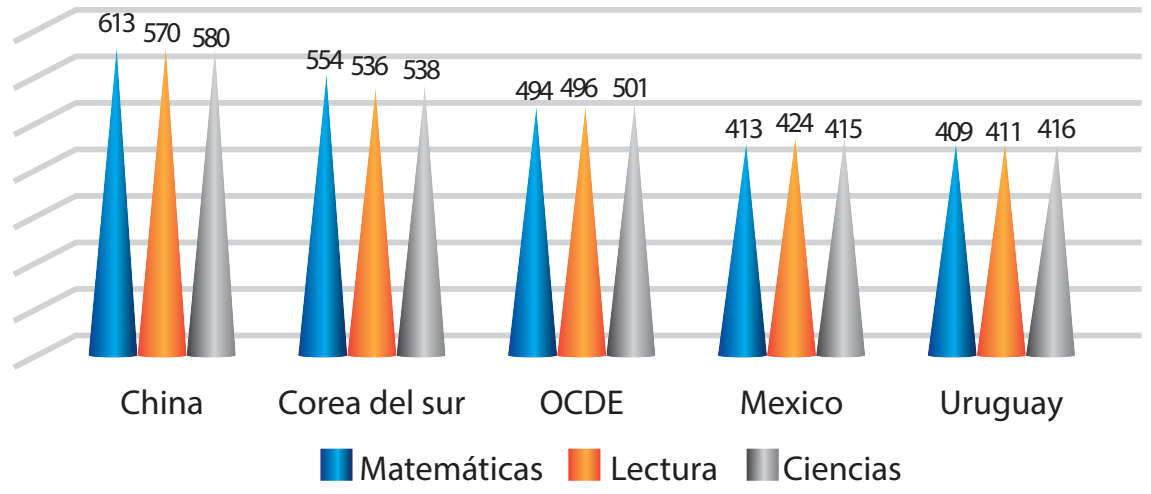

Figura 2. Gráfico comparativo de resultados prueba PISA 2012. Elaboración propia con base en Pisa (2013).

Uruguay registró un estancamiento en los últimos años, por su parte México "muestra un avance lento, pero progresivo, que tardarían dos generaciones para alcanzar los niveles de la OCDE" (Montt, 2015, párr. 14). Esta dinámica se ve reflejada en el último reporte de The Global Information Technology Report 2014, donde México cayó 16 posiciones ubicándose en el lugar 79. Los altos costos de los servicios de internet (lugar 93), el bajo nivel educativo (lugar 119) y la poca presencia de empresas con capacidad de innovar (lugar 75) son los indicadores que no han podido mejorarse (The Global Information Technology Report, 2014). La dinámica de innovación dentro del sector del software se ha visto afectada por las recomendaciones de los clientes (learning by using) que, en los últimos años, se han convertido en pieza fundamental para la mejora de software y plataformas. México y China han tenido un importante avance en las conexiones a internet en los últimos años; sin embargo, Uruguay y La República de Corea desde los noventas ya tenían la infraestructura necesaria. Esta condición permite que la sociedad pueda ser un mercado para las empresas locales de software y un laboratorio para el desarrollo de nuevas aplicaciones. 


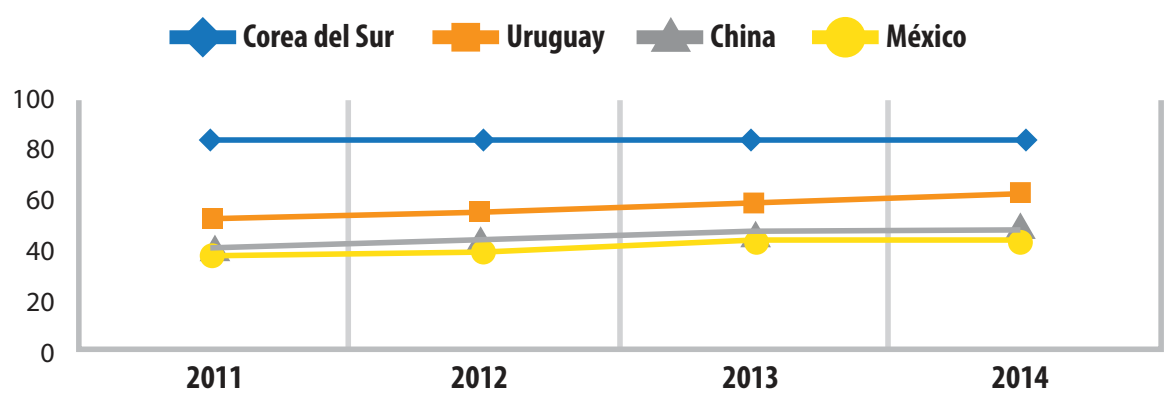

Figura 3. Usuarios de internet por cada 100 habitantes.

Elaboración propia con base en el Banco Mundial (2015).

Uno de los pilares fundamentales para transitar a una economía del conocimiento es la inversión destinada a la investigación y desarrollo (I+D). Los países asiáticos destinan más del 1\% y la República de Corea ha logrado pasar el $3 \%$. Por su parte, Uruguay y México no han podido superar el 1\%.

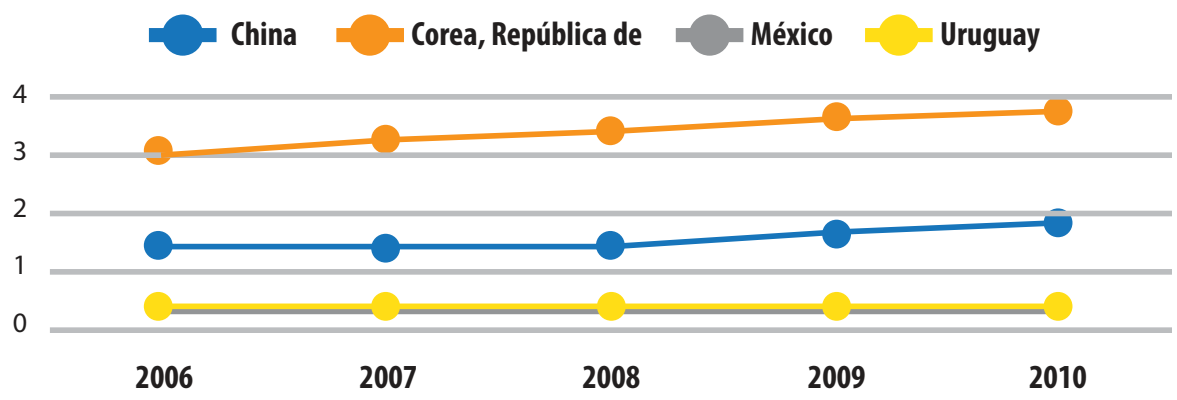

Figura 4. Porcentaje del PIB dedicado a I+D.

Elaboración propia con base en el Banco Mundial (2015).

Parte importante del crecimiento del sector del software es el uso de nuevas tecnologías y sistemas en los gobiernos; por lo que la transformación y la demanda que exijan los gobiernos son fundamentales para que las empresas de software desarrollen plataformas. China y La República de Corea le han apostado al fortalecimiento del mercado interno para transformar sus industrias, sobre todo transitar al e-government que permita desarrollar softwares locales; así evitan el pago de licencias a compañías extranjeras. Uruguay ha presentado 
grandes avances en los últimos años. Según United Nations e-government survey (2014), Uruguay ha subido 24 posiciones del 2012 al 2014, pasando del 50 al 26 con un total de 0.7420 . Se ha colocado en cuarto lugar en e-participation por arriba de Japón, Francia y Estados Unidos (ONU, 2014); es considerado uno de los diez países con mayor crecimiento del 2012 al 2014. La República de Corea lleva una década en los primeros cinco lugares, logrando la mayor calificación en e-government value.

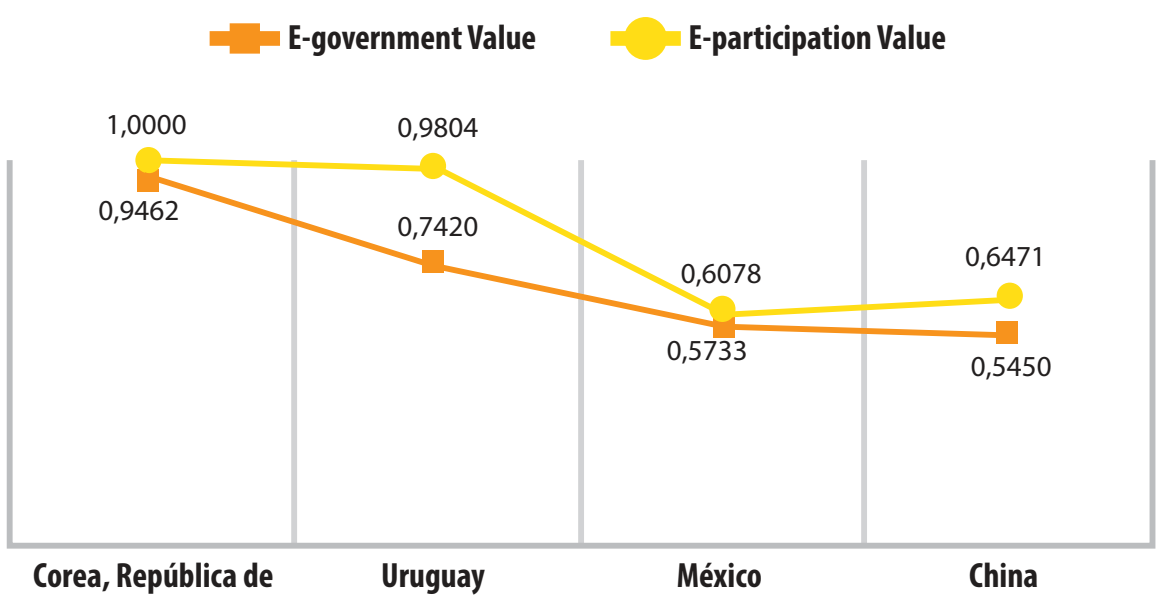

Figura 5. Comparativo en e-government y e-participation, 2014.

Elaboración propia con base en el UNPACS (2015).

\section{El mercado internacional de software y sus implicaciones}

Parte esencial del desarrollo del sector del software se ve embebido por el mercado mundial de software y el desarrollo de aparatos tecnológicos; por tal razón, los países tienen que implementar un sistema de software para mejorar sus capacidades de innovación y desarrollo. Esta capacidad del software "es la clave para la participación en la economía global del conocimiento" (Tessler, Barr y Hanna, 2003, p. 3), ya que la dinámica del mercado global de software es altamente competitiva e internacionalizada.

En la actualidad, una empresa de la India puede subcontratar a una empresa en México para el desarrollo de software de un cliente ubicado en Estados Unidos; o una empresa puede tener living labs alrededor del mundo con un grupo de Ingenieros que desarrolla software. El software, por su facilidad de distribución, ha facilitado 


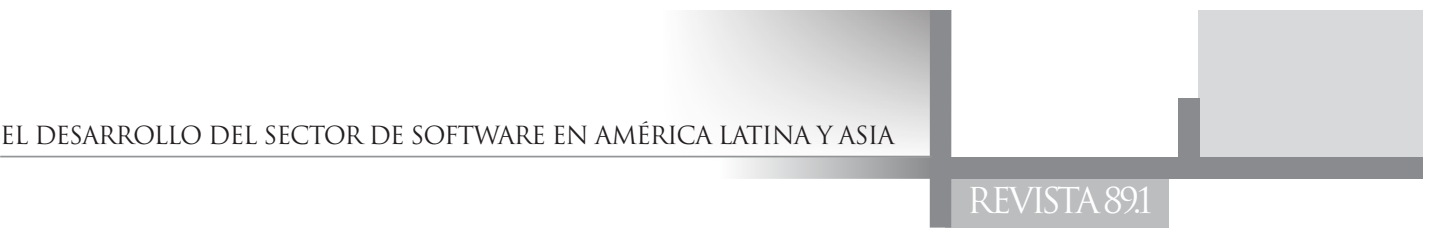

que empresas internacionales vendan software a diferentes gobiernos y a empresas trasnacionales que llevan su paquete de software a todas sus filiales.

Las alianzas en el sector de software son cada vez más comunes para enfrentar más rápido el mercado global y contar con oficinas para el soporte técnico. Ante este panorama, las alianzas estratégicas del sector del software son parte fundamental para entender la dinámica que modifica el mercado y las formas de competencias. El caso más emblemático es Microsoft, con alianzas con diversas empresas de computadoras portátiles para preinstalar su Office; además de su alianza con Blaupunkt para el uso de Windows CE Automotive, que hoy utilizan FORD, Fiat, Nissan y Kia (Sainz, 2014; Windows, 2015). El sector bancario y el de telefonía, en los últimos años, han creado alianzas con las empresas de software para la reducción de costos, el manejo de tráfico de llamadas, socios comerciales, plataformas y desarrollo de software para los sistemas.

Internacionalmente, una de las alianzas que puede modificar el mercado global de productos de alta tecnología (HT) es la relación China-India, donde China produciría todos los aparatos electrónicos e India los servicios y programas para que funcionen. Si se fortalece esta relación, América Latina encontraría una competencia muy fuerte y la inversión en nuevas tecnologías se daría en asociación con empresas chinas e indias para la modernización tecnológica, fortaleciendo así la cooperación académica y la investigación conjunta de desarrollo; o los países que no cuentan con las condiciones para desarrollar el sector del software solo compran tecnología con los desarrollos y servicios de software.

Esta dinámica global obliga a los países a diversificar sus economías y buscar exportar más bienes manufacturados de alto valor agregado; el desarrollo del sector del software es, por tanto, una vía para el desarrollo de América Latina (OCDE, 2014). "Ahora es el momento de crear las condiciones y tomar la iniciativa. Lo que es más, no hay otra alternativa” (OCDE, 2014, párr. 11).

Según la consultora Gartner, el sector del software alcanzó los 407,3 mil millones de dólares. Esto implica un incremento de 4.8 puntos porcentuales con respecto a las ventas de 2012, que ascendieron a 388.5 mil millones de dólares (Diario TI, 2015). En términos económicos, solo la renovación de licencias a nivel mundial representó 423 billones de dólares en 2012 (PwC Global, 2013). Por su parte, el mercado de software en América Latina en 2014 cerró con un valor de mercado de 13,305 millones de dólares, lo que representó un crecimiento de $12.8 \%$ en contraste con el 17.2\% registrado de 2013 (Posada, 2015).

En la actualidad, la tendencia del sector del software es la creación de nubes dentro de la internet, lo que implica resolver la reconversión misma de la empresa; esto es, 
pasar de ser una empresa desarrolladora de software a la medida o en paquete, a una que produce y vende servicios donde no es el software ni el desarrollo de software lo que se vende, sino un servicio digital donde la nube se relaciona con servidores desde internet, encargados de atender las peticiones en cualquier momento. Implica una reconfiguración de la industria para crear y mantener las nubes con los datos y aplicaciones necesarias de los clientes y de cambiar los software desarrollados al lenguaje de la nube, lo cual conlleva a una modificación en el proceso de creación. La forma de comercializar el software dentro de la nube obliga a las industrias especializadas a adaptarse y modificarse para mantenerse en el mercado, debido a que no vende en sí el producto, vende licencias de uso a diversas empresas.

La renovación de licencias y la creación de software as a service (SaaS) han modificado la dinámica del mercado global de software, hoy se busca desarrollar software que sea adaptado y no desarrollos individuales. En este rubro América Latina creció un 18\% en el desarrollo de software de soporte a la integración y orquestación de aplicaciones (Posada, 2015).

Por último en "la economía del conocimiento está presente la confusión entre conocimiento e información; esto se debe a que en la nueva economía del conocimiento los trabajadores tienen que tomar decisiones por medio de elecciones racionales basada en información" (Lundvall y Lorenz, 2010, p. 47). Por tal razón, la economía del conocimiento se centra en la habilidad de codificar, manipular y transmitir gran cantidad de información a un bajo costo con ayuda de las tecnologías de la información (TIC).

A raíz de ello surge la creciente demanda de aplicaciones y soluciones integrales para los big data y la entrega de análisis en el menor tiempo; aunado a esto, el resguardo de información, instalación y mantenimiento de las redes de comunicación, manejo de los sistemas instalados en las empresas, capacitación, etc. Son servicios que han crecido a la par de los big datas y porque grandes empresas han deslocalizado los servicios TIC. "No tiene caso que yo tenga 20 servidores que implican altos costos de mantenimiento, cuando con un clic me puedo conectar a países como Estados Unidos y Australia, en donde puedo rentar servidores" (Buitrón, en Durán, 2014, párr. 5). Por tanto, los países tienen que crear condiciones de seguridad e infraestructura para resguardar grandes cantidades de información y convertirse en opciones internacionales para recibir servidores.

A pesar de ser considerado un sector altamente innovador y de nuevas formas de organizar el trabajo que crea desarrollo, el sector del software no está fuera de la dinámica de mercado. La división del trabajo ha sido parte fundamental del desarrollo de diversos sectores industriales, el caso del sector del software 


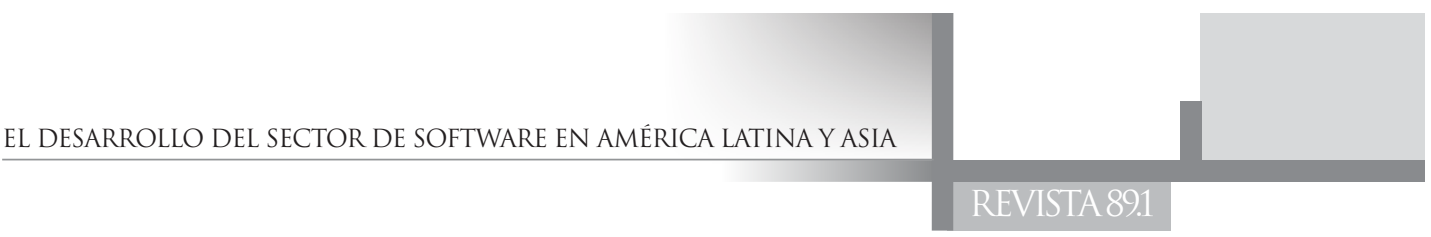

no es la excepción, ya que busca reducir costos y tiempo para la creación de un software; los costos se reducen mediante la tercerialización de procesos de baja categoría.

En el estudio realizado en la ciudad de Querétaro, México, las empresas trasnacionales de software instaladas en la ciudad no generan vinculación con las empresas locales y no tienen un impacto en las empresas del entorno local. Para entender esto, se tiene que partir de que las empresas trasnacionales instaladas en la entidad no pueden modificar el software por la cuestión de derechos de autor y todo desarrollo que se da está protegido por la empresa, no se puede modificar o compartir; además, las empresas trasnacionales de software requieren de empresas certificadas, y con capacidades técnicas y humanas para desarrollar software en conjunto o puede darse el servicio de testing.

En los últimos años se han encontrado dinámicas de monopolios que lo ejercen aquellas empresas que tienen a la venta un producto o servicio único gracias a la tecnología que se ha empleado en su desarrollo que, además, está respaldado por una patente que da garantía de que no se reproduzca el conocimiento sin antes pagar regalías al desarrollador. Esta dinámica se genera de igual forma en los socios comerciales de software, que a pesar de tener una relación comercial, no pueden modificar el software. Otra forma de controlar el software es el estar programados en un código, lo que dificulta la exportación a otros lenguajes.

En el caso del sector del software, se comercializa el producto y la aplicación que despliega, no el saber hacer. En la distribución del software es indiferente la manufactura, porque la reproducción en grandes cantidades es irrelevante, puesto que el costo de replicar un programa es prácticamente nulo, "lo importante aquí es el diseño, el desarrollo y, lo más importante, su utilidad" (Márquez, 2007, p. 65). En la ciudad de Querétaro se identificaron islas de innovación dentro de las empresas trasnacionales instaladas en la entidad que, a través de grupos de trabajos virtuales, desarrollan software y recurren al outsourcing de las empresas locales solo para la programación del software, el mantenimiento, venta del software o licencias o soporte técnico. Estas situaciones complejizan el mercado internacional de software y permiten ampliar las discusiones sobre el desarrollo del sector del software a través de alianzas con empresas locales, ya que se rompen con las limitantes del espacio geográfico y del desarrollo del sector de software a partir de islas de innovación en diversos espacios geográficos.

Además, se han dado prácticas poco leales en el sector del software. Se encuentran los casos de Huawei que, en Estados Unidos, fue considerado una amenaza para la seguridad Nacional (El País, 2012); sin embargo, Huawei en 2015 lanzó el Huawei P8 lite. Por su cuenta, Tata Consultancy Services 
enfrenta una investigación del Departamento del Trabajo estadounidense por una violación de las reglas de visas para los trabajadores extranjeros en el sector de TIC (Jopson y Crabtree, 2015); debido a que Tata Consultancy Services ha solicitado 5650 visas de trabajo; aunque siete empresas que tienen base en India han solicitado 16573 visas (Park, 2015).

El caso del crecimiento de China en las exportaciones de alta tecnología (HT) tuvo un impacto negativo en América Latina. "China exportó \$375 billones de dólares y América latina exportó 67 billones de dólares" (Gallagher y Porzecanski, 2008, p. 12). México es el país más amenazado, "el $97 \%$ de las exportaciones de alta tecnología de México están bajo alguna amenaza de China...... los subsectores de HT que son los más amenazados son máquinas de oficina, computadoras, televisores, equipos de telecomunicaciones, aparatos de electricidad y maquinaria eléctrica" (Gallagher y Porzecanski, 2008, p. 15). Por otra parte, la manufactura de equipos de telecomunicación y sus accesorios ha sido afectada por la incursión de las empresas chinas en el mercado de los Estados Unidos de Norteamérica, de 2000 a 2006 México decreció 6.8\% sus exportaciones y China aumentó el 23.0\% (Gallagher y Porzecanski, 2011).

\section{Exportaciones de software}

La inserción al mercado internacional de software se puede dar bajo tres vías: a) como un receptor de proyectos de innovación y desarrollo, b) brindando servicios de software para el mercado creciente de TIC en el mundo, o c) desarrollando software con empresas nacionales para competir en el mercado internacional. La inserción al mercado global de software se vislumbra de forma positiva, cuando un sector de software está consolidado y puede desarrollar software conservando la licencia y los derechos del software. La apuesta por el mercado doméstico es, quizá, la más importante para el desarrollo del sector de software en economías emergentes (Tessler S, Barr A. y Hanna N, 2003).

Para la consolidación del sector de las TIC, China es un claro ejemplo de apostarle al mercado local y crear una política de protección y desarrollo de tecnologías de empresas chinas. "Los logros de China a este respecto son destacables. Según estadísticas oficiales de China, la producción nacional de software pasó de 7.000 millones de dólares en 2000 a 285, 000 millones de dólares en 2011. Se calcula que cerca del $90 \%$ de esa producción está destinada al mercado interno, aunque por lo general se integra en la fabricación de productos de TIC, y de otros productos, que son luego exportados" (ONU, 2012, p. xv). 
China ha ingresado al mercado internacional de software por medio de compañías nacionales que brindan diversos servicios de software. Neusoft (especializado en e-hospital) y Yonyou, se encuentran en 71 y 99 lugar respectivamente en el top 100 de compañías de software. Pero en la actualidad China cuenta con tres empresas más que tienen gran impacto internacional.

Tabla 1.

Empresas de China con renombre internacional

\begin{tabular}{|l|l|}
\hline \multicolumn{1}{|c|}{ Empresa } & \multicolumn{1}{c|}{ Reseña } \\
\hline Yonyou & $\begin{array}{l}\text { Reconocida como un proveedor líder de software de gestión empre- } \\
\text { sarial y de servicio en la nube en Asia Pacífico. Con sede en Pekín, } \\
\text { Yonyou. Cuenta con } 100 \text { oficinas y se extiende por el mundo con más } \\
\text { de } 12.000 \text { empleados. }\end{array}$ \\
\hline Chinasoft & $\begin{array}{l}\text { Empezó con proyectos para e-government en las cinco zonas de creci- } \\
\text { miento (Tianjin, Dalian, Harbin, Beijing and Guangzhou) para el Mi- } \\
\text { nisterio de Transporte, de Agricultura y para el de Desarrollo Urbano- } \\
\text { Rural. En la actualidad continúa con los proyectos de e-government, } \\
\text { proporcionando los servicios de la nube para el municipio de Zhejiang, } \\
\text { cuenta con 27. 847 empleados }\end{array}$ \\
\hline Huawei & $\begin{array}{l}\text { Se está consolidando como primer vendedor de smartphones en China, } \\
\text { desplazando a Samsung y Apple. A nivel internacional está en tercer } \\
\text { lugar detrás de Samsung y Apple. }\end{array}$ \\
\hline Xiaomi & $\begin{array}{l}\text { Se ha colocado detrás de Huawei en ventas internacionales de } \\
\text { smartphones. }\end{array}$ \\
\hline
\end{tabular}

Nota: Elaboración propia con base en páginas oficiales de las empresas.

El crecimiento de estas empresas chinas es similar, partieron del desarrollo de productos para el mercado interno y tuvieron un impulso por parte del gobierno federal. Las empresas de software han optado por tener una inversión mixta y hacer de sus trabajadores accionistas de las empresas; lo que ha logrado una mayor identificación y que el personal realice una carrera dentro de las empresas conservando el conocimiento. Esto ha ocasionado que en la dinámica de China se haga difícil conocer la separación entre lo público y lo privado (Studwell, 2011). Estas grandes empresas asiáticas encuentran grandes ventajas en los países en desarrollo, convirtiéndose en obstáculos para las empresas locales que no cuentan con los recursos económicos y de infraestructura, para emprender un desarrollo de varios años. 
La República de Corea tiene el sector de TIC más grande por unidad PIB (UNCTAD, 2012). La República de Corea no se caracterizaba por las exportaciones de software, fue en 2010 cuando el gobierno lanzó el programa Korea Quantum Jump Strategy, lo que permitió aumentar de 6 billones de dólares en 2008 a 14 billones de dólares en 2013. Una de las estrategias planteadas fue la vinculación de las empresas de software con las manufactureras, empresas coreanas como Daewoo, Samsung, Hyundai, Kia Motors y LG tienen desarrollo de software para los electrodomésticos, carros y dispositivos de comunicación; además, el gobierno apoyo abrir los códigos para generar innovación abierta y activas de Saas (UNCAT, 2012).

La empresa de software Hancom desarrolló el procesador para el lenguaje nativo de Corea, Hangul; es una de las pocas compañías a nivel mundial que ha rechazado la compra de Microsoft. Hancom desarrolló Hancom Office como competencia de office y el gobierno coreano, en apoyo a la empresa, compra las licencias de software de Hancom, lo cual lo convierte en el primer gobierno que no compra servicios de Microsoft. A la par, en el ámbito de videojuegos, está Nexon Corporation y Nc soft que se han colocado entre el top 100 de empresas de software a nivel mundial.

Por su parte, Uruguay no desarrolla software como estos países asiáticos, pero ha dado un importante paso en la consolidación de e-government. Uruguay muestra una vía diferente al desarrollo por el mercado local. "Las exportaciones de software y servicios de TI superan ampliamente los gastos en el interior del país por los mismos conceptos, mientras que el gasto interno en software es relativamente bajo" (UNCAT, 2012, párr. 10). Una de las explicaciones a este fenómeno es la configuración del territorio uruguayo: las empresas tienen que nacer pensando en la exportación, para lo cual el mercado interno es como un laboratorio de su producto o servicio para después exportar (Cabrera, 2014).

En Uruguay aproximadamente existen 550 empresas en la industria TIC, de acuerdo con la clasificación por actividad principal que realiza la Cámara Uruguaya de Tecnologías de la Información (CUTI, 2014). El éxito de Uruguay se debe a su exportación mundial y su impacto en el mercado de los Estados Unidos; su dinámica exportadora le permite competir en un mercado amplio y desarrollar software constantemente para mantenerse en la vanguardia. Esto le permite sobrellevar las crisis de diferentes países, diversificar los clientes y mantener un desarrollo continuo. El último reporte de la CUTI manifiesta que las exportaciones de TIC en 2011 llegaron a 266 millones de dólares, destacándose como principales destinos de los productos y servicios uruguayos: Estados Unidos (26,4\%), Brasil (12,42\%), Chile, (9,3\%), Argentina (8,5\%) y México (8,29\%) (CUTI, 2012). 
La mayor ventaja que ofrece Uruguay al sector es la capacidad técnica de sus recursos humanos gracias a su temprano desarrollo y a las instituciones educativas que cuentan con un cúmulo de conocimientos, que permiten replantear los planes de estudio y enfrentar las nuevas problemáticas.

Por otra parte, existen diversas empresas uruguayas que se han consolidado a través de los años, porque han podido mantener el conocimiento dentro de ellas, lo que permite la actualización del software y generar una marca propia. Uruguay cuenta con GENEXUS que se logró posicionar en el mercado con su software GENEXUS por los conflictos sociales en Israel; convirtiendo su software en el más utilizado a nivel mundial para la programación. La crisis del 2002 les enseñó a diversificar los productos y mercados internacionales para no depender de un solo cliente, lo cual forzó a crear una dinámica exportadora y la colocación de diversas empresas uruguayas en el exterior, así como la creación de marcas de software de Uruguay. El fuerte del desarrollo de software a la medida o en paquete es para el extranjero, concentrándose en: $i$ ) software financiero, ii) de gestión orientado a Pymes, iii) software de desarrollo orientado a programadores y $i v$ ) software verticales que consiste en desarrollo de software a empresas trasnacionales (López Beneites, 2006).

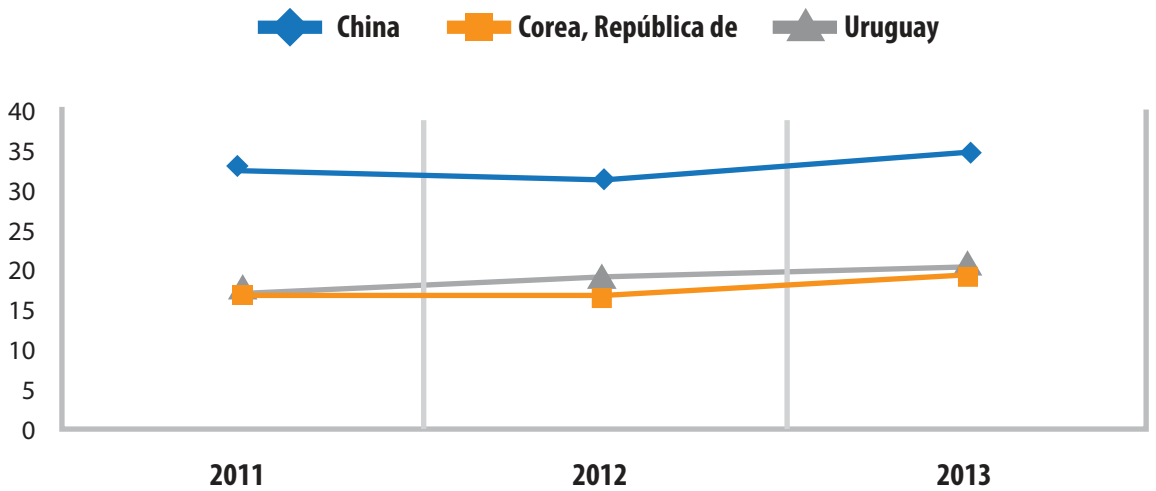

Figura 6. Porcentaje de exportaciones de Servicios de TIC.

Elaboración propia con base en el Banco Mundial, 2015.

México aún se encuentra desarrollando al sector de las TIC con el programa de Plataforma Digital, donde busca consolidar al sector del software creando una base de datos de las empresas desarrolladoras en México para la programación de software en conjunto. Por lo que se han destinado grandes recursos para el fortalecimiento del sector de software. PROSOFT, dependencia federal encargada del desarrollo de TIC, destinó en 2014722 millones de pesos, basada 
en la proyección de la política para el 2018 por medio de la Estrategia Digital Nacional conocida como México Digital que busca: i) la conectividad, ii) la inclusión de habilidades digitales, iii) interoperatividad, iv) datos abiertos y v) marco jurídico. Ha alineado las estrategias del PROSOFT para tener una economía digital, la transformación del Estado, el uso de las TIC para garantizar a la ciudadanía la seguridad y la salud; y el uso de estas para mejorar la educación e insertarse con éxito en la sociedad de la información y el conocimiento (Estrategia Digital Nacional, 2013).

La gran inversión extranjera directa (IED) en diversos sectores económicos (automotriz, aeronáutico, compañías de desarrollo de soluciones y compañías proveedoras de tecnología de marca) le permitirán a México tener clientes potenciales. De acuerdo con Kearney, México ocupó el segundo lugar en América Latina como destino de inversión de proyectos de software, atrayendo $23 \%$ de la inversión total; además, es considerado el mejor destino de América para el establecimiento de compañías de TIC, Esto convierte a México en el lugar primordial en América Latina, debido a su razonable costo y a la buena-educación de los trabajadores (The 2014 A.T Kearney Global Services Location Index, 2014 y PROSOFT, 2015).

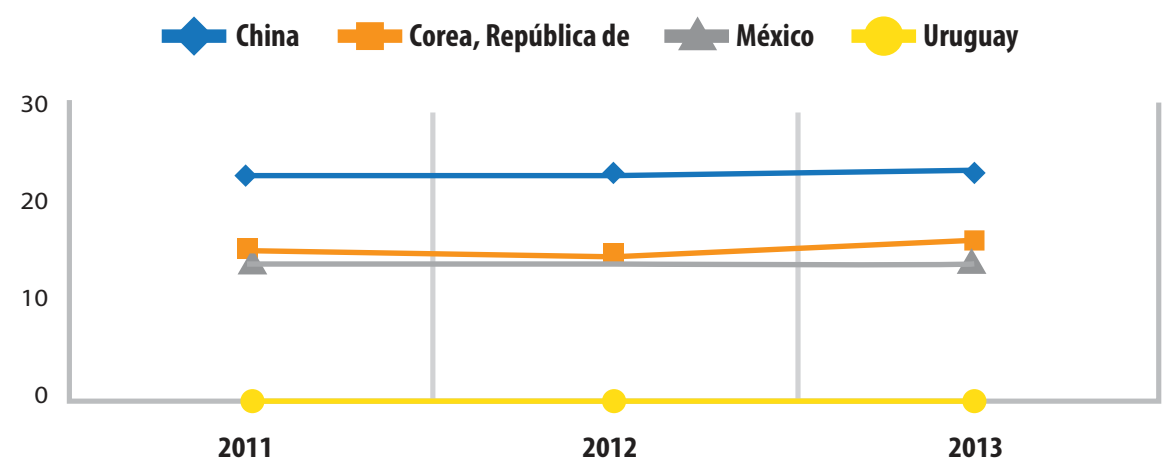

Figura 7. Exportaciones de productos de TIC (\% de las exportaciones de productos). Elaboración propia en base al Banco Mundial, 2015.

El crecimiento del sector de software en México se da, en gran medida, por actores particulares que crean fondos de crédito o han logrado posicionarse en el mercado de software de los Estados Unidos de Norteamérica. Casos como Icazar, Claudio Cossio, Santiago Zavala y César Salazar, entre otros, han puesto en alto los desarrollos en el sector TIC a nivel mundial. Existen actualmente diversas plataformas para apoyar la creación de startups mexicanas: Santiago 
Zavala y César Salazar crearon Mexican VC, primer fondo de capital de riesgo que presta dinero a emprendedores para convertir sus ideas en herramientas útiles y tangibles (Calderón, 2014).

En México y Uruguay se empieza a debatir diferentes formas de financiar proyectos de TIC y la forma de medir el riesgo y el impacto para otorgar los apoyos económicos a las empresas de software; si bien los dos países no tienen un sistema de innovación consolidado y una gran cartera de software, en Uruguay la CUTI y en México el CNCS ofrecen, en sus páginas web, los software y productos de las empresas afiliadas.

\section{Conclusiones}

México y Uruguay han tenido un crecimiento en los últimos años, pero no les ha permitido alcanzar los niveles de la OCDE y consolidar un sector de software. Los indicadores de educación y de inversión en investigación (I+D) son muy bajos para los países latinoamericanos; caso contrario, los países asiáticos que han aumentado el nivel educativo y la inversión en I+D en las últimas décadas.

La república de Corea y China son ejemplos de que la inversión privada y la gubernamental no son excluyentes para el desarrollo de sectores industriales. De igual forma, el desarrollo de otros sectores industriales permite crear un mercado al sector del software y nuevas exigencias que ayudan a que el software sea competitivo en el mercado global. Por ejemplo, China, con el desarrollo de smartphones, y la República de Corea, con el desarrollo de autos, electrodomésticos, computadoras, laptops, tabletas y tecnologías de la comunicación.

Para el desarrollo del sector del software, el mercado interno es parte importante, debido a que ayuda a las empresas a adquirir los conocimientos prácticos necesarios y crear nuevos productos para competir a nivel global. En la actualidad, las empresas asiáticas están ingresando al mercado creando una oportunidad de crecimiento para las empresas locales de software, por medio de alianzas para participar conjuntamente en investigaciones, modernización tecnológica o una cooperación académica; no obstante, también puede crear una barrera para el desarrollo de empresas locales. Esta situación pone a los países en dos caminos para desarrollar el sector del software: a) la inversión y apoyo a empresas locales para que desarrollen software y puedan desarrollarse sin condiciones de tiempo o económicas; o $b$ ) construir alianzas y colaboraciones con empresas trasnacionales para encontrar un punto de apoyo en el mercado local que permita involucrarse en las tendencias globales y, posteriormente, incursionar en el mercado global de software (Mingzhi y Ming, 2003). 
ENERO - JUNIO - 2016 ROLANDO J. SALINAS G. ' DANIEL MONTES P.

REVISTA 891

Por último, "es muy importante que desde ahora las empresas que venden en el extranjero instrumenten medidas que les den certeza sobre la legalidad de sus instrumentos informáticos para asegurar sus estrategias de exportación" (Tsuru, 2013, p. 21). El sector de las TIC crece cada año y con apoyo gubernamental o sin él tiene que encontrar la forma de desarrollarse y crear las condiciones para consolidarse. Por tanto, cada año se hace más apremiante la regulación del mercado global y nacional de software que dé certeza al sector de software; de igual forma se tienen que desarrollar las leyes de protección de derechos de autor, el régimen tributario y los avances del derecho informático, de manera que permita tener una solvencia y reglas establecidas en el mercado interno tanto para empresas nacionales como extranjeras. 


\section{Referencias}

ARNeT. (Febrero, 2014). POLICY BRIEF BRIEF, NO. 38. Recuperado de http:// www.unescap.org/sites/default/files/polbrief38.pdf

Calderón, Verónica. (2014). El boom tecnológico mexicano. Reuperado de http://tecnologia.elpais.com/tecnologia/2014/10/22/actualidad/1414011317_572594.html

Casas y Dettmer. (2013). Sociedad del conocimiento, capital intelectual y organizaciones innovadoras. En Giovanna Valente y Mónica Casalet, Instituciones, sociedad del conocimiento y mundo de trabajo. FLACSO.

CUTI. (2012). Encuesta Anual de CUTI. Recuperado de http://www.cuti.org.uy/ novedades/2819-la-industria-tics-de-uruguay-crecio-un-22.html

CUTI. (2014). Acerca de Cuti. Recuperado de http://www.cuti.org.uy/acerca-decuti.html

Diario TI. (2014). El mercado global del software creció en $4.8 \%$ en 2013. Diario TI. Recuperado de http://diarioti.com/el-mercado-global-del-software-crecio-en-4-8-en-2013/76725

Gallagher and Porzecanski. (2011). Clear and Present Danger: Mexico and China in the U.S. Market (Chapter 5). En The Dragon in the Room. Stanford University Press.

Gallagher and Porzecanski. (2011). The State of the State: Industrial Reform in Mexico and China (Chapter 6 and 7). En The Dragon in the Room. Stanford University Press.

Durán, Lourdes. (2014). Augura InteQsoft tendencias de TIC. Financiero. Recuperado de http://eleconomista.com.mx/estados/2014/03/16/augura-inteqsoft$\underline{\text { tendencias-tic }}$

Jopson y Crabtree (2015). US probes Infosys and Tata Consultancy over H1-B visas. Recuperado de http://www.ft.com/intl/cms/s/0/a7196022-1115-11e59bf8-00144feabdc0.html\#axzz42NMji3Qt

Kearney's Global Service Location Index. (2011). Recuperado de http://www. atkearney.com/documents/10192/f062cfd8-ee98-4312-ae4f-0439afc10880

Lundvall, Bengt y Lorenz, Edward. (2010). Innovación y desarrollo de competencias en la economía del aprendizaje. Implicación para las políticas de innovación (Parte 1). En M.Parrilli (Coord.), Innovación y aprendizaje (pp. 45-102) España: Orkestra. 
Márquez, M. (2007). Ingenieros rancheros: Elección cultural y estilo tecnológico. México: Plaza y Valdez.

MONTT, G. (2015). No sorprende el resultado de Uruguay en las pruebas PISA. En El país. Recuperado de: http://www.elpais.com.uy/informacion/no-sorprende-resultado-uruguay-pruebas.html

Networked Readiness Index 2015. (2015). Report Highlights. World Economic (Forum). Recuperado de http://reports.weforum.org/global-information-technology-report-2015/report-highlights/\#heatmap

OCDE. (2014). The impact of china and india in latinamerica, challenges and opportunities. Recuperado de: http://www.oecd.org/dev/theimpactofchinaandindiainlatinamericachallengesandopportunities.htm . Consultado el 05/11/2015

ONU. (2014). UNITED NATIONS E-GOVERNMENT SURVEY . Recuperado de 2014 http://unpan3.un.org/egovkb/Portals/egovkb/Documents/un/2014Survey/E-Gov_Complete_Survey-2014.pdf

Park, H. (2015). How Outsourcing Companies Are Gaming the Visa System. The Ney York Times. Recuperado de: http://www.nytimes.com/ interactive/2015/11/06/us/outsourcing-companies-dominate-h1b-visas.

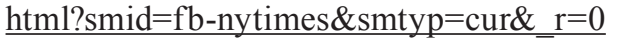

Posada, M. (17, septiembre, 2015). Se redujo crecimiento del mercado de software en $A L$ durante 2014. Recuperado de: http://www.jornada.unam.mx/ ultimas/2015/09/17/se-redujo-crecimiento-del-mercado-de-software-en-alen-2014-5662.html.

Sainz, J. (2014). Alianzas estratégicas en la práctica. Madrid: ESIC.

Studwell, J. (2011). Where China Fits. In: How Asia Works (Chapter 4). Profile Books.

Tsuru, K. (Enero - febrero, 2013). Software legal y exportación a Estados Unidos. Comercio Exterior, 63(1), Bancomext.

T-Kearney global Rewards and Risks of Big Data. (2014). Recuperado de: https://www.atkearney.com/gbpc/global-services-location-index/past-report/-/asset_publisher/i9mYsV84tcQx/content/offshoring-opportunitiesamid-economic-turbulence-the-a-t-kearney-global-services-location-index-2011/10192?_101_INSTANCE_i9mYsV84tcQx_redirect $=\% 2$ Fresearchstudies $\% 2$ Fglobal-services-location-index

The 2014 ATKEarney Global Services Location Index. (2014). A wealth of choises: from anywhere on earth to no location at all. Recuperado de http://www. basis.org.bd/resource/A\%20Wealth\%20of\%20Choices.pdf 
Tessler, S., Barr, A., \& Hanna, N. (2003). National software industry development: considerations for government planners. EJISDC: The Electronic Journal on Information Systems in Developing Countries, 13(9), City University of Hong Kong.

UNCAPS. (2015). Rankin Internacional de e-government. Recuperado de: ghttp:// unpan3.un.org/egovkb/en-us/Data-Center

UNCAT. (2012a). Según la UNCTAD se abren nuevas posibilidades para la industria local del software en los países en desarrollo. Recuperado de: http:// unctad.org/es/Paginas/PressRelease.aspx?OriginalVersionID=109

UNCAT . (2012b). INFORMATION ECONOMY REPORT 2012: The Software Industry and Developing Countries.

Windows. (2015). Sistemas embebidos Windows 7. Recuperado de: http://www. microsoft.com/windowsembedded/en-us/windows-embedded-automotive-7.aspx.

\section{Entrevistas:}

Cabrera, G. (2014) Ejecutivo de Nuevos Emprendimientos y Fuentes de Financiamiento de CUTI. Entrevistado en la ciudad de Montevideo, Uruguay el 15/10/2014. 